\title{
Protocol for a Randomized Phase II Trial for Mesh Optimization by Autologous Plasma Coating in Prolapse Repair: IDEAL Stage 3
}

\author{
Holger Gerullis · Dimitri Barski · Evangelos Georgas · Mihaly Borós • \\ Albert Ramon · Thorsten H. Ecke - Silvia Selinski · Dörte Luedders • \\ Mario W. Kramer · Alexander Winter · Friedhelm Wawroschek • \\ Thomas Otto
}

Received: January 9, 2017 / Published online: February 23, 2017

(C) Springer Healthcare 2017

\section{ABSTRACT}

Introduction: Mesh-related complications especially after vaginal implantation have raised awareness lately because of severe adverse reactions and legal aspects. About 20\% of patients suffer from complications after mesh insertion in the anterior vaginal wall. Autologous plasma coating of meshes prior to implantation has shown potential to improve the biocompatibility of meshes in vivo and in vitro. This inno-

Enhanced content To view enhanced content for this article go to http://www.medengine.com/Redeem/ ED87F060514E549F.

H. Gerullis · D. Barski · E. Georgas · T. Otto Department of Urology, Lukas Hospital Neuss, Neuss, Germany

H. Gerullis $(\bowtie) \cdot$ A. Winter · F. Wawroschek University Hospital for Urology, Klinikum Oldenburg, School of Medicine and Health Sciences, Carl von Ossietzky University, Oldenburg, Germany e-mail: holger.gerullis@gmx.net

H. Gerullis · D. Barski · T. H. Ecke

The IDEAL collaboration, Oxford, UK

M. Borós

Department of Experimental Surgery, University of Szeged, Szeged, Hungary

\section{A. Ramon}

International Tissue Engineering Research

Association (ITERA), Antwerp, Belgium vative approach has been developed according to the IDEAL recommendations for surgical innovations. The method has still to be assessed at stage 3 accordingly.

Methods: A protocol is developed for a prospective single-blinded randomized controlled phase II trial for biocompatibility optimization of anterior vaginal meshes for prolapse repair by autologous plasma coating versus non-coated meshes.

Results: The protocol aims at fulfilling the requirements for stage 3 (assessment) according to IDEAL. Eligible for inclusion are women with primary cystocele, requiring a surgical procedure, suitable for randomization, and willing to be randomized. Participants will be followed up

T. H. Ecke

Department of Urology, HELIOS Hospital, Bad Saarow, Germany

S. Selinski

Leibniz Research Centre for Working Environment and Human Factors, IfADo, Dortmund, Germany

D. Luedders

University Hospital for Gynecology, Klinikum Oldenburg, School of Medicine and Health Sciences, Carl von Ossietzky University, Oldenburg, Germany

M. W. Kramer

Department of Urology, University of Luebeck, Luebeck, Germany 
by postal questionnaires ( 6 months post surgery and 12 months post randomization) and will also be reviewed in clinic 12 and 24 months post surgery. Primary endpoint is the assessment of mesh-related complications following the Clavien-Dindo classifications. QoL, sexual function assessment, efficacy, and validation of an already developed long-term register are considered secondary endpoints. To afford a calculated $10 \%$ reduction of postoperative complications through plasma-coated meshes vs. non-coated meshes at 1-year follow-up, a total 214 women in each arm will be necessary to achieve $80 \%$ power at a significance level of $5 \%$.

Conclusion: The protocol for this randomized clinical trial represents the conditions to assess the surgical innovation of plasma coating of meshes in order to improve the meshes' biocompatibility at stage 3 according to the IDEAL recommendations.

Keywords: Clinical trial; IDEAL recommendations; Plasma coating; Prolapse repair; Quality of life; Surgical innovation; Surgical mesh; Vaginal prolapse

\section{INTRODUCTION}

The Innovation, Development, Exploration, Assessment, and Long-term study (IDEAL) recommendations were first introduced in 2009 by McCulloch et al. to increase the evidence and transparency requirements for surgical innovations during their entire development and learning process. The IDEAL recommendations suggest what types of studies, approaches, and reporting should be used for surgical innovations and procedures. Table 1 gives an overview of the different IDEAL stages and the respective requirements [1-3]. The criteria were extended in 2016 to IDEAL-D for medical devices to improve product surveillance, even at preclinical stages of development, in order to rationalize regulatory structures and to minimize regulatory delay [4].

During recent years the development of alloplastic materials and respective US Food and Drug Administration (FDA) warnings concerning high complication rates associated with transvaginal placement of surgical meshes to treat pelvic organ prolapse (POP) and stress urinary incontinence (SUI) have lead to the conviction that a detailed and differentiated evaluation of mesh application is needed. However, the question of whether traditional or prosthetic repair of cystocele should be preferred is still under discussion and not yet backed up with sufficient and clear data when balancing best clinical results to lowest complication rates [5]. Regardless meshes have been increasingly used in this indication. After two official FDA warnings in 2008 and 2011 an upgrade in risk classification for transvaginal meshes was demanded, allowing the request of premarket approval and postmarket surveillance studies [6]. Finally, in 2016 the FDA changed the risk class from II to high risk III for vaginal mesh for POP treatment [7]. For meshes currently used in surgeries for hernia, incontinence (SUI), and prolapse (POP) conditions, respectively, the optimization of biocompatibility features has gained increasing focus in clinical and preclinical research and sophisticated methods have been developed [8, 9]. However, none of those approaches have progressed beyond experimental stages and were therefore not further investigated or applied in humans. In addition, none of those innovations have been conducted, labeled, or interpreted according to the IDEAL recommendations. Their potential to be implemented into clinical use is critical, from both a scientific and regulatory point of view.

In a very recent publication, Sedrakyan et al. discussed the potential of the recommendations for better structuring evaluation and regulation of medical devices [4]. In particular implantable devices have been identified as helpful tools for many patients. Those devices have also, unfortunately, led to serious adverse problems in multiple occasions [10-12]. Regulatory authorities in both the USA and Europe are proceeding towards stricter requirements for new devices to be approved. The FDA now requires a pivotal clinical trial through its premarket approval or within the $510(\mathrm{k})$ pathway for vaginal mesh implants. In Europe, the evolving medical device reform (MDR) will set comparable standards. 
Table 1 IDEAL stages, respective requirements, and already concluded preliminary work according to IDEAL-D within this project

\begin{tabular}{|c|c|c|c|c|}
\hline IDEAL stage & Label & Primary outcome & Study design & Reference \\
\hline 0 & Preclinical & Concept, safety & $\begin{array}{l}\text { Experimental studies } \\
\text { (animal, cadaver) }\end{array}$ & {$[15-18]$} \\
\hline 1: Idea & "First in human" & Innovation & $\begin{array}{l}\text { Case report, case series, } \\
\text { registration }\end{array}$ & {$[21]$} \\
\hline $\begin{array}{l}\text { 2: } \\
\text { Development }\end{array}$ & $\begin{array}{l}\text { "Tinkering" of device, } \\
\text { few adopters }\end{array}$ & Development, safety, efficacy & Prospective cohort trials & {$[21]$} \\
\hline 3: Assessment & Assessment & Compare to standard & $\begin{array}{l}\text { Randomized controlled } \\
\text { trial or similar }\end{array}$ & $\begin{array}{l}\text { Current } \\
\text { protocol }\end{array}$ \\
\hline 4: Long term & $\begin{array}{l}\text { Registry, long-term } \\
\text { evaluation }\end{array}$ & $\begin{array}{l}\text { Quality assurance, identification of risk } \\
\text { factors, comparators }\end{array}$ & Registry & {$[22]$} \\
\hline
\end{tabular}

For reaching the requirements of stage 3 for medical devices, in particular implantables and/ or substantial modifications, a randomized controlled trial showing clinical efficacy would normally be required [13]. However, the IDEAL recommendations offer alternative experimental approaches to reach stage 3 level, e.g., tracker trials, adaptive designs, and economic modeling-based studies [4, 14].

Our group has shown in preliminary studies over 4 years that mesh modification by coating with autologous plasma results in improved biocompatibility performances. Those findings were shown to be consistent under both in vivo and in vitro conditions [15-18].

In contrast to other researchers covering this experimental field, from the very beginning our entire experimental approach has been conducted by following the five-step IDEAL system for surgical innovations. The project therefore remains comparable and reproducible at every single step of development and represents a unique process of development for a medical device modification with clinical potential $[19,20]$.

As a consequent next step this experimental approach of mesh coating with autologous plasma was transferred into human conditions where the procedure proved to be safe, feasible, and reproducible [21]. According to the assessment stage requirements a prospective randomized trial comparing the effect of plasma coating to non-coated meshes would be the next step in the development process.

Additionally, in order to provide the appropriate infrastructure to fulfill stage 4 (long-term study) our group has already developed a consensus based online registry for registration and outcome measurement of implant-assisted operations for POP and female and male SUI. This registry has already been tested for feasibility [22].

In this current research paper we present a protocol for a prospective single-blinded randomized controlled trial for biocompatibility optimization of mesh by autologous plasma coating. This trial protocol is adapted to the SPIRIT recommendations for interventional trials [23]. The chosen indication is mesh application to the anterior vaginal wall for the treatment of POP. The primary goal is to investigate mesh-related complications of plasma-coated meshes compared to naïve, noncoated meshes. Secondary study goals are QoL assessment and clinical efficacy for POP treatment.

\section{METHODS}

The presented method of mesh coating with autologous plasma is based on a German Patent (DE Patent: 102011000 666.4). The current study protocol is based on the feasibility 
analysis of an already concluded successful pilot study with 20 patients [21]. Application of autologous blood plasma coating is performed according to the German Pharmaceutical Law (AMG), the Medical Product Act (MPG), and the Transfusion Act. In both study centers at least one investigator will hold the necessary official regulatory requirements to conduct clinical trials according to both AMG and MPG.

\section{Surgical Procedure}

In this study we have chosen the indication of anterior vaginal wall prolapse, i.e., mainly cystocele (prolapse of the anterior vaginal wall) but also combined urethrocele (urethra prolapse) or paravaginal defect (pelvic fascia defect). Reportedly, in vaginal surgery, the most common recurrence site is the anterior vaginal wall with reported failure rates of about $30 \%$. Therefore alloplastic materials have been and are widely used in this indication to improve the durability of POP repair and to reduce the rate of recurrence [24]. The indication and associated mesh use represent an appropriate possibility to investigate our approach of plasma-coated meshes. In this trial, women will get surgical correction of a cystocele via anterior vaginal wall repair with an alloplastic mesh. We will use a commonly available alloplastic mesh material, which has been previously tested with plasma coating in preliminary animal studies (PVDF material). The particular mesh will be defined before implementing the trial and will be used in all participating study centers.

\section{Plasma Coating}

Prior to the implantation the modified meshes will be incubated for at least $30 \mathrm{~min}$ with 20-40 $\mathrm{ml}$ autologous plasma, which is obtained from the respective patient by vein puncture before anesthesia induction. The underlying surgical technique is not altered by the application of our technology and the operation time will be increased by a maximum of $5 \mathrm{~min}$ as shown in a previous pilot study [21]. Figure 1 gives a simplified illustration of the two main steps of the procedure.

\section{Protocol Development}

A protocol for a prospective single-blinded randomized controlled phase II trial for biocompatibility optimization of anterior vaginal meshes for prolapse repair by autologous plasma coating has been developed in collaboration with the participating institutions. The protocol has been created according to the CONORT recommendations (http://www. consort-statement.org). It has been evaluated and adapted to the SPIRIT (Standard Protocol Items Recommendations for Interventional Trials) 2013 Checklist (http://www.spiritstatement.org/wp-content/uploads/2013/01/ SPIRIT-Checklist-download-8Jan13.pdf). A CONSORT diagram is provided for the workflow of this clinical trial (Fig. 2).

\section{RESULTS}

\section{Study Population}

In general, this clinical trial will include women with anterior wall prolapse requiring corrective surgery (POPQ $\geq \mathrm{III})$. Potential candidates will be identified by their treating office-based gynecologists and urologists and referred to one of the study centers. Eligible women who declare an interest in participating in the trial will receive a patient information leaflet describing the study and informing about the scientific background, previous results, etc. They will also be supplied with a baseline questionnaire and a consent form as well as an information sheet concerning the respective study participation insurance. All patients will sign an informed consent for randomization and in order to use their data for the study. Once those documents are received, every woman will get a personal study number.
(a) Inclusion
criteria:
Female
patients $>18$ years old, affected by $\geq 3$ stage POP, according to the Pelvic Organ 

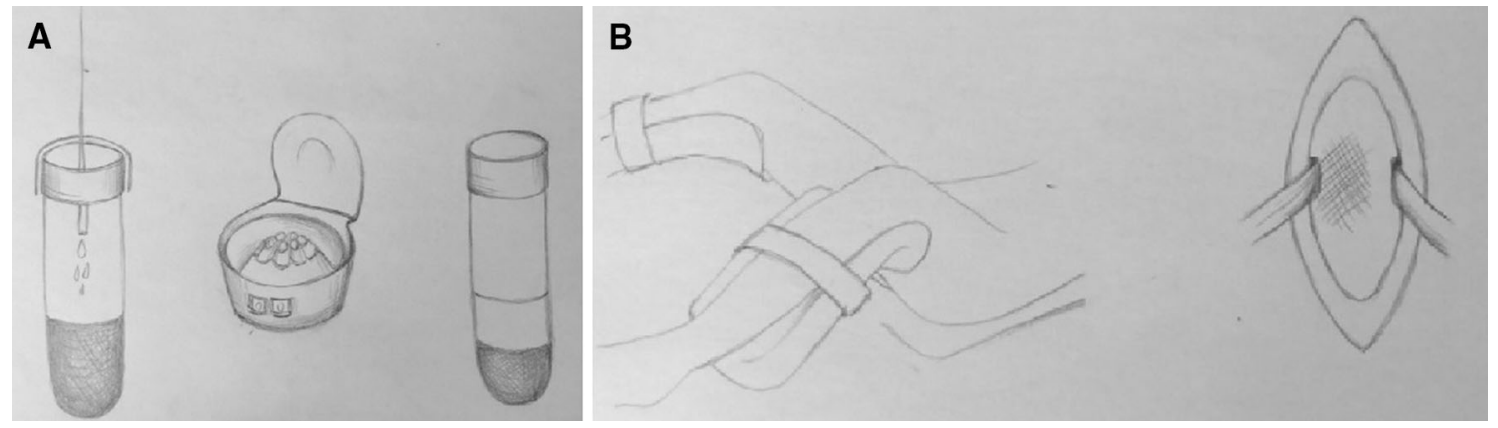

Fig. 1 Mesh coating and application. Plasma retrieval from all patients' blood probes (a) and mesh insertion with autologous plasma or native mesh after randomization $(\mathbf{b})$

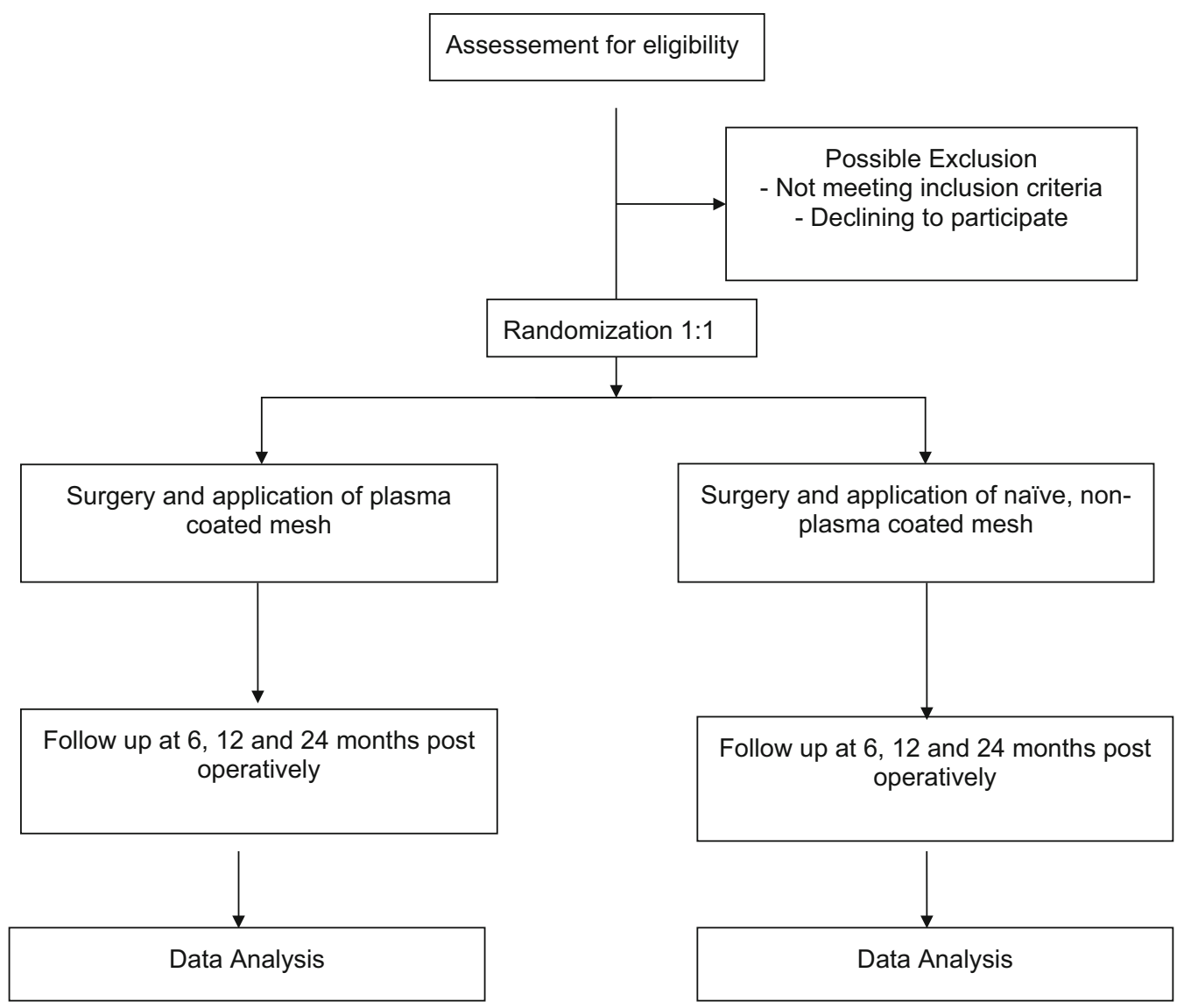

Fig. 2 CONSORT flow diagram for current trial

Prolapse Quantification system (POP-Q) classification (score 0-4), indication for anterior vaginal repair (mesh use), patients able and willing to complete at least 24 months of follow-up. Must be suitable and willing for being randomized. (b) Exclusion criteria: Patients with previous prolapse surgery, hysterectomy, vaginal surgery or radiotherapy. Comorbidities representing contraindications for major surgery. Comorbidities demanding specific different surgical approach (e.g., fibroids, scarring/adhesions). Other exclusion 
criteria: potential future pregnancies, infection, chemo- or immunological therapy during the last 3 months, colpocleisis, obesity, psychiatric illness and pregnancy, reduced compliance and inability to complete at least 24 months of follow-up.

\section{Study Objectives and Endpoints}

\section{Primary Endpoint}

- Assessment of mesh-related complications (erosion, infection, dyspareunia, mesh shrinkage, chronic pelvic pain $>6$ weeks) using the Clavien-Dindo classification [25]. Aim is to answer the question: does plasma coating of meshes prior to implantation lead to improved tolerance?

\section{Secondary Endpoints}

- Quality of life assessment [use of validated questionnaires (POP-QoL, ICIQ-SF-2004)].

- Sexual function assessment [use of the validated German translation of the Pelvic Organ Prolapse/Incontinence Sexual Questionnaire IUGA Revised (PISQ-IR)] [26, 27].

- Assessment of functional results with regard to repair of prolapse, incontinence pelvic floor sonography and vaginal examination, efficacy assessed by objective and subjective cure rates, for POP defined as absence of vaginal bulge symptoms, SUI defined as negative stress test and absence of self-reported SUI symptoms (POP-Q questionnaire, ICIQ-SF 2004 questionnaire) [28, 29].

- Validation of the already created register by inscription of participating patients [22].

\section{Outcome Measurement}

The clinical follow-up will be performed during the hospital stay at 6,12 , and 24 months postoperatively.

The complications are followed in accordance with the Clavien-Dindo classification of surgical complications. Mesh-associated complications are classified according to Clavien-Dindo and ICS/IUGA definition and include exposure or erosion of mesh, local infection or fistula, and pelvic pain ( $>6$ weeks)
[29]. Mesh erosion is defined as exposed or extruded mesh material in the vagina or surrounding pelvic organs. Intraoperative, early (from discharge and less than 6 weeks), and delayed complications are differentiated. We consider the absence of more than grade 2 and/ or any "delayed" Clavien-Dindo complications to define success.

Before surgery, all patients will provide a detailed case history and completed a questionnaire on their urinary and POP symptoms. The standardized and internationally validated incontinence questionnaire ICIQ-SF (International Consultation on Incontinence Questionnaire-Short Form) as a measure for the psychological strain of incontinence will be obtained from the patients during the first visit. The score ranges from zero to 21 , with zero being no strain at all.

The standardized and internationally validated P-QOL score is used to assess POP symptoms. Ratings between 0 and 100 are used to measure the severity of vaginal prolapse. A higher score indicates a greater impairment of quality of life than a lower score. The domain and symptom questions have individual scores and are not combined with a whole questionnaire score [30].

Patients undergo a clinical urogynecological examination, a pelvic ultrasound scan (to exclude uterine or ovarian disease), and a vaginal inspection in the gynecological and standing positions at rest and under maximum straining (cough test) with a full bladder for SUI. The women are examined in supine position using the Pelvic Organ Prolapse Quantification (POP-Q) system of the International Continence Society (ICS) as a validated and reliable method to classify a prolapse [31].

\section{Randomization and Blinding}

This study cannot be double-blinded since coating of the mesh is performed in the operating room prior to implantation of the mesh. Therefore the study is single-blinded to the patient. A 1:1 randomization is chosen; allocation into the two study groups will be completely randomized using the program 
www.randomizer.at. This randomization application will be available as an Internet-based service. Randomization will be carried out as close to the time of surgery as is possible.

\section{Statistical Methods and Sample Size Considerations}

In current literature, the complication rate after mesh-assisted vaginal prolapse repair is assumed to be about $20 \%$ according to published studies and meta-analyses [28, 32]. In accordance with experimental and pilot trials we expect a reduction of mesh-related postoperative complications to $10 \%$ at 1 -year follow-up by autologous plasma-coating of mesh prior to implantation. A total 214 women in each arm would be required to achieve $80 \%$ power at a significance level of 5\%. To test the hypothesis, a two-sided $\chi^{2}$ test is used. On the basis of experiences in comparable trials, a realistic estimated percentage of patients lost to follow-up would be $15 \%$. It is planned to actively stay in contact with the patients (telephoning, appointments, etc.) in order to reduce this number. However, allowing for those $15 \%$ lost to follow-up at 1 year would require 252 women to be recruited to each arm (504 in total).

Both participating centers have a high reputation in the field and regularly perform high volumes of mesh-applying surgeries in this indication. The planned and already concluded advertisement strategies include presentation of the trial at local scientific meetings, presentations at loco-regional hospitals, etc. We assume a steady state recruitment rate of approximately 3 women per month per center, i.e., a total of 72 patients per year. However, considering wavering recruitment numbers possibly related to risk factors, patient preferences, contraindications for mesh implantations, etc., we calculated a realistic recruitment period of 4 years.

\section{Number of Centers Involved}

We aim to recruit women from two high-volume centers with experience of over 70 POP surgeries per year and extended scientific research in this field. Those centers are the
Pelvic Floor Centre of the Lukas Hospital Neuss, Department of Urology, Neuss Germany and the University Hospital for Urology, Klinikum Oldenburg, School of Medicine and Health Sciences, Carl von Ossietzky University Oldenburg. The study will be conducted in collaboration with the DZITM (German Centre for Assessment and Evaluation of Innovative Techniques in Medicine). Both original centers will have at least one surgeon with at least 10 years of experience in prolapse surgery with a minimum of 50 procedures per year. Both centers collaborate with regional gynecological centers to ensure that at least one additional surgeon with equal experience level is available and to increase the recruitment pool. In case of significant delay in recruitment the protocol offers the possibility to be amended in terms of inclusion of additional center(s). However, center(s) with potential to be added have to show corresponding quantity and experience in this surgical indication and need to satisfy all requirements demanded in this protocol.

\section{Documentation and Data Handling}

Outcome assessment is achieved via participant self-completed questionnaires, which helps to avoid an interviewer bias. Documentation of protocol-required information will be performed via standardized case report forms which will be reviewed and signed by the investigator or an appropriate subinvestigator. All data will be transferred pseudonymously. Patients and research staff will not be explicitly informed if the applied mesh was randomly plasma coated or not during the surgical procedure. It will not be possible to get this information via examination features, etc., either for the patient or for the physician involved in the follow-up. In cases of necessary explantation immunohistochemistry analyses and in vitro biocompatibility tests of the explanted mesh will be performed [15].

All participating patients names and respective confidential information are subject to medical confidentiality under the German Data Protection Act. Transmission of data will be 
done in an encrypted format. No access to the original documents will be given to others not involved in the trial. Both patient and physician will sign the informed consent to obtain and validate it. Patients can quit participation in the trial at any time without disclosure of their motives. In this case all relevant data will be deleted if desired by the patient.

\section{Trial Registration}

This trial has been registered at Research Registry ${ }^{\circledR}$ as researchregistry1991 (http://www.researchre gistry.com/browse-the-registry.html\#home/regi strationdetails/58597cb0ff66105645672327/).

Research Registry ${ }^{\circledR}$ is a new online-based free of cost registry platform launched in 2015 [33]. It is in accordance with the recommendations and allows prospective and retrospective data registration. This register offers major advantages like simplicity; it is free for all users to register and it features open access. Researchers get immediate visibility of registered projects.

\section{Ethical Issues}

This trial will be performed according to the Declaration of Helsinki (DoH) for ethical principles in human beings [34]. The study protocol will get an ethical vote from the Carl von Ossietzky University, Oldenburg, Germany and subsequently a vote from the Ethics Commission of Nordrhein Westphalen representing the regional official institutions of both study centers. Although there is no expected increased risk for patients with plasma-coated meshes, we will provide a proband insurance for all participants of the clinical trial. This article does not contain any new studies with human or animal subjects performed by any of the authors.

\section{Funding}

Potential institutions will be addressed subsequently to publishing the protocol.

\section{DISCUSSION}

On the basis of extended in vitro and in vivo preliminary results we hypothesized that autologous plasma coating of meshes prior to implantation improves the mesh's biocompatibility. It should therefore result in a lower mesh-related complication rate in the intervention group compared to the patient group with naïve meshes. The entire preliminary experimental approach has been strictly performed following the IDEAL recommendations for surgical innovations and our group has already implemented an online-based registry as stage 4 (long-term study). In conclusion, this study has two paramount aims: (1) to investigate the hypothesis of improved biocompatibility of plasma-coated meshes compared to naive meshes and (2) to fulfill the final and missing stage 3 (assessment stage) of this novel method of mesh plasma coating.

When aiming to complete the development process of a surgical innovation according to the IDEAL recommendations a clinical trial (preferably randomized in nature) is necessary to fulfill the requirements for the assessment stage. In this particular project we have already been able to develop an online-based registry providing with the required tool for the longterm study. Thus, by publishing a study protocol for assessing autologous plasma coating of meshes in the specific indication of POP surgery all IDEAL stages will be displayed and completed for this innovative surgical approach. It will therefore be the first reported surgical innovation which has been visualized according to IDEAL at every step of development [20].

Appropriate trial registration is another important issue for this study protocol. We have chosen Research Registry ${ }^{\circledR} \quad$ (www. researchregistry.com). This registry is free of charge and very easy to handle. In addition, it features the main reporting recommendations. The registry had reportedly received 500 registrations by September 2016 [33]. It represents a valuable tool to officially visualize study protocols even when funding/sponsoring are not confirmed yet as is the case in this current 
protocol. Chapman and colleagues reported that one in five surgical randomized controlled trials (RCT) are discontinued early, and one in three will not be published 2 years after trial conclusion [35]. Easy and smart trial registration tools are important to avoid redundancy and to help visualizing protocols in order to prespecify research questions and outcomes and, last but not least, to document protocols even when trials cannot be conducted or concluded for unforeseeable reasons (recruitment problems etc.).

Choice of the appropriate measurement tool for QoL after POP surgery can be challenging. In this protocol the assessment of sexual function will be done with the Pelvic Organ Prolapse/ Incontinence Sexual Questionnaire IUGA Revised (PISQ-IR) which is the most appropriate questionnaire and even offers a validated German translation [26, 27]. Being composed of 12 different items, three main factor groups are displayed in this questionnaire: the behavioral emotive factor (4 items), the physical factor (5 items), and the partner-related factor score (3 items). In a recent study, Vitale and co-workers successfully used this tool to evaluate sexual function after cystocele repair with porcine mesh graft in 20 women and proved it to be a feasible tool in this indication [36]. For QoL measurement they use the Short Form-36 (SF-36) validated questionnaire which poses 36 questions and is grouped into eight assessed categories [37]. It represents a very easy to use tool for QoL assessment. However, in this protocol we have chosen the POP-QoL, ICS SF-2004 for QoL assessment which has also been validated in German and is easy to answer and commonly used [30].

The paramount strength of this protocol is the amount of already available supporting data on the experimental method of plasma coating for meshes and it's positive effect on biocompatibilty. Several groups have developed coating strategies of meshes used in prolapse surgery. Mesh coating with collagen has been mainly investigated in animal studies showing positive effects on biocompatibility [38-41]. However, this approach has not shown convincing results for the treatment of anterior vaginal wall prolapse when applying it in humans [42, 43].
Other techniques like coating with highly purified collagen or fetal collagen both in a rat model $[44,45]$ and platelet-enriched plasma in a rabbit model [46] have shown promising biocompatibility improvements in vivo. Even a study using a macaques model for the investigation of extracellular matrix regenerative graft reported that regenerative extracellular matrix graft attenuated the negative impact of polypropylene on the vagina [47]. However, despite promising, mainly comparative studies in animals no randomized trial in humans has been initiated so far to compare any of those coating strategies to non-coated meshes.

Besides in vitro and in vivo data, the method has already proven feasible in a pilot study with 20 patients, thereby diminishing safety concerns [21]. In addition, our group has already developed and successfully implemented a long-term register for meshes and mesh modifications [22]. Thus, according to IDEAL, the method needs to be assessed, most preferably in an RCT setting. Another strength is that this trial can be easily randomized and single-blinded to the patient. Previous data show that there is no increased risk when applying meshes which have been previously coated with autologous plasma.

This study protocol has limitations. An important limitation is the high number of required patients ( $n=214$ not including calculated lost to follow-up patients) per randomization arm which is necessary to prove (or not) the main outcome goal of decreased mesh-related complications when using plasma-coated meshes. As this study is bicentric in nature and although both centers are high-volume institutions, recruiting 428 or even 504 POP patients over 4 years is likely to be very difficult. A statistical advantage can only be shown given this data. Therefore, trial conclusion could be difficult recruiting-wise as the number of calculated patients is high. However, inclusion of additional centers by amendment is possible according to the protocol. An important dilemma of RCT in surgical innovations becomes visible: practicability may be unrealistic when statistical advantages are the only criteria to assess a surgical innovation. The IDEAL recommendations are aware of this difficult 
situation and provide alternative designs to fulfill stage 3 requirements (observational studies, interrupted time series) [48]. However, when evaluating the technique of plasma coating of meshes an RCT is technically feasible as described.

Another limiting factor for the success of this trial is the challenge of finding appropriate funding sources or even financial supporters for an RCT with a modification of an already approved medical product. However, after successful publication of an approved trial protocol, institution funds and public funds will be targeted.

One can imagine that this trial could show problems with regard to recruitment or funding. However, in this particular project it is of outmost importance that all IDEAL stages are covered and published accordingly. Thus, it assures awareness of necessary completion of all IDEAL stages for the surgical innovation of autologous plasma coating of meshes in order to evaluate its potential in broad clinical application.

\section{CONCLUSIONS}

The protocol for this randomized clinical trial represents the conditions to assess the surgical innovation of plasma coating of meshes in order to improve the meshes' biocompatibility at stage 3 according to the recommendations.

\section{ACKNOWLEDGEMENTS}

No funding or sponsorship was received for this study or publication of this article.

All authors read and approved the final manuscript. All named authors meet the International Committee of Medical Journal Editors (ICMJE) criteria for authorship for this manuscript, take responsibility for the integrity of the work as a whole, and have given final approval for the version to be published. Holger Gerullis and Dimitri Barski contributed equally to this manuscript.

Disclosures. Holger Gerullis, Dimitri Barski, Evangelos Georgas, Mihaly Borós, Albert
Ramon, Thorsten H. Ecke, Silvia Selinski, Dörte Luedders, Mario W. Kramer, Alexander Winter, Friedhelm Wawroschek, and Thomas Otto have nothing to disclose.

Compliance with Ethics Guidelines. This trial will be performed according to the Declaration of Helsinki. The protocol will be approved by the regional ethical commissions of the participating centers and possible additional centers. The study will only start once those institutions have given their positive vote. This article does not contain any new studies with human or animal subjects performed by any of the authors.

Data Availability. The datasets during and/ or analyzed during the current study are available from the corresponding author on reasonable request.

\section{REFERENCES}

1. McCulloch P, Altman DG, Campbell WB, et al. No surgical innovation without evaluation: the IDEAL recommendations. Lancet. 2009;374(9695): 1105-12. doi:10.1016/S0140-6736(09)61116-8.

2. Ergina PL, Cook JA, Blazeby JM, et al. Challenges in evaluating surgical innovation. Lancet. 2009; 374(9695):1097-104. doi:10.1016/S0140-6736(09) 61086-2.

3. Barkun JS, Aronson JK, Feldman LS, et al. Evaluation and stages of surgical innovations. Lancet. 2009;374(9695):1089-96.

4. Sedrakyan A, Campbell B, Merino JG, Kuntz R, Hirst A, McCulloch P. IDEAL-D: a rational framework for evaluating and regulating the use of medical devices. BMJ. 2016;353:i2372.

5. Vitale SG, Lagana AS, Gulino FA, Tropea A, Tarda S. Prosthetic surgery versus native tissue repair of cystocele: literature review. Updates Surg. 2016;68(4):325-9. doi:10.1007/s13304-015-0343-y.

6. UPDATE on serious complications associated with transvaginal placement of surgical mesh for pelvic organ prolapse: FDA safety communication hwfgMSAuh. https://www.fda.gov/downloads/ MedicalDevices/Safety/AlertsandNotices/UCM2627 60.pdf. Accessed 1 Jan 2017. 
7. FDA Gastroenterology-Urology Medical Devices Advisory Committee Panel. Reclassification of orogynecologic surgical mesh instrumentation. http://www.fda.gov/downloads/AdvisoryCommitte es/CommitteesMeetingMaterials/MedicalDevices/ MedicalDevicesAdvisoryCommittee/Gastroentero logy-UrologyDevicesPanel/UCM487224.pdf. Accessed 1 Jan 2017.

8. Junge $\mathrm{K}$, Rosch R, Klinge U, et al. Titanium coating of a polypropylene mesh for hernia repair: effect on biocompatibilty. Hernia. 2005;9(2):115-9.

9. Lukasiewicz A, Skopinska-Wisniewska J, Marszalek A, Molski S, Drewa T. Collagen/Polypropylene composite mesh biocompatibility in abdominal wall reconstruction. Plast Reconstr Surg. 2013; 131(5):731e-40e. doi:10.1097/PRS.0b013e31828 $65 \mathrm{~d} 2 \mathrm{c}$.

10. Sedrakyan A. Metal-on-metal failures-in science, regulation, and policy. Lancet. 2012;379(9822): 1174-6. doi:10.1016/S0140-6736(12)60372-9.

11. Chatterjee S, Herrmann HC, Wilensky RL, et al. Safety and procedural success of left atrial appendage exclusion with the lariat device: a systematic review of published reports and analytic Review of the FDA MAUDE Database. JAMA Intern Med. 2015;175(7):1104-9.

12. Kramer DB, Xu S, Kesselheim AS. How does medical device regulation perform in the United States and the European Union? A systematic review. PLoS Med. 2012;9(7):e1001276.

13. Smith AJ, Dieppe P, Vernon K, Porter M, Blom AW, National Joint Registry of E, et al. Failure rates of stemmed metal-on-metal hip replacements: analysis of data from the National Joint Registry of England and Wales. Lancet. 2012;379(9822):1199-204.

14. Lilford RJ, Braunholtz DA, Greenhalgh R, Edwards SJ. Trials and fast changing technologies: the case for tracker studies. BMJ. 2000;320(7226):43-6.

15. Gerullis H, Georgas E, Eimer C, et al. Evaluation of biocompatibility of alloplastic materials: development of a tissue culture in vitro test system. Surg Technol Int. 2011;21:21-7.

16. Gerullis $H$, Klosterhalfen $B$, Boros $M$, et al. IDEAL in meshes for prolapse, urinary incontinence, and hernia repair. Surg Innov. 2013;20(5):502-8.

17. Gerullis H, Georgas E, Eimer C, et al. Coating with autologous plasma improves biocompatibility of mesh grafts in vitro: development stage of a surgical innovation. Biomed Res Int. 2013;2013:536814.

18. Gerullis H, Georgas E, Boros M, et al. Inflammatory reaction as determinant of foreign body reaction is an early and susceptible event after mesh implantation. Biomed Res Int. 2014;2014:510807.

19. McCulloch P. The IDEAL recommendations and urological innovation. World J Urol. 2011;29(3):331-6.

20. Gerullis H, Barski D, Ecke TH, et al. Autologous plasma coating improves the biocompatibility of mesh implants. On the IDEAL way from bench to bedside. Int J Surg. 2016; 36(Suppl 2):S142. doi:10. 1016/j.ijsu.2016.11.050.

21. Barski D, Gerullis H, Georgas E, et al. Coating of mesh grafts for prolapse and urinary incontinence repair with autologous plasma: exploration stage of a surgical innovation. Biomed Res Int. 2014;2014:296498. doi:10.1155/2014/296498.

22. Barski D, Gerullis H, Ecke TH, et al. In: Register of urogynecological implants: the development of an online platform for registration and outcome measurement according to the IDEAL long-term stage of surgical innovation. Int J Surg 2016;36(Suppl 2):S141-2. doi:10.1016/j.ijsu.2016.11.048.

23. Chan AW, Tetzlaff JM, Altman DG, et al. SPIRIT 2013 statement: defining standard protocol items for clinical trials. Ann Intern Med. 2013;158(3):200-7.

24. Olsen AL, Smith VJ, Bergstrom JO, Colling JC, Clark AL. Epidemiology of surgically managed pelvic organ prolapse and urinary incontinence. Obstet Gynecol. 1997;89(4):501-6.

25. Dindo D, Demartines N, Clavien PA. Classification of surgical complications: a new proposal with evaluation in a cohort of 6336 patients and results of a survey. Ann Surg. 2004;240(2):205-13.

26. Rogers RG, Rockwood TH, Constantine ML, et al. A new measure of sexual function in women with pelvic floor disorders (PFD): the Pelvic Organ Prolapse/Incontinence Sexual Questionnaire, IUGA-Revised (PISQ-IR). Int Urogynecol J. 2013;24(7):1091-103.

27. Trutnovsky G, Nagele E, Ulrich D, et al. German translation and validation of the Pelvic Organ Prolapse/Incontinence Sexual Questionnaire-IUGA revised (PISQ-IR). Int Urogynecol J. 2016;27(8):1235-44.

28. Barski D, Otto T, Gerullis H. Systematic review and classification of complications after anterior, posterior, apical, and total vaginal mesh implantation for prolapse repair. Surg Technol Int. 2014;XXIV:217-24.

29. Haylen BT, Freeman RM, Swift SE, et al. An International Urogynecological Association (IUGA)/ 
International Continence Society (ICS) joint terminology and classification of the complications related directly to the insertion of prostheses (meshes, implants, tapes) and grafts in female pelvic floor surgery. Neurourol Urodyn. 2011;30(1):2-12.

30. Lenz F, Stammer H, Brocker K, Rak M, Scherg H, Sohn C. Validation of a German version of the P-QOL Questionnaire. Int Urogynecol J Pelvic Floor Dysfunct. 2009;20(6):641-9.

31. Bump RC, Mattiasson A, Bo K, et al. The standardization of terminology of female pelvic organ prolapse and pelvic floor dysfunction. Am J Obstet Gynecol. 1996;175(1):10-7.

32. Maher C, Feiner B, Baessler K, Christmann-Schmid C, Haya N, Marjoribanks J. Transvaginal mesh or grafts compared with native tissue repair for vaginal prolapse. Cochrane Database Syst Rev. 2016;2:CD012079.

33. Agha R, Fowler AJ, Limb C, et al. The first 500 registrations to the Research Registry ${ }^{\circledR}$ : advancing registration of under-registered study types. Front Surg. 2016;3:50.

34. World Medical Association. World Medical Association Declaration of Helsinki: ethical principles for medical research involving human subjects. JAMA. 2013;310(20):2191-4.

35. Chapman SJ, Shelton B, Mahmood H, Fitzgerald JE, Harrison EM, Bhangu A. Discontinuation and non-publication of surgical randomised controlled trials: observational study. BMJ. 2014;349:g6870.

36. Vitale SG, Caruso S, Rapisarda AM, et al. Biocompatible porcine dermis graft to treat severe cystocele: impact on quality of life and sexuality. Arch Gynecol Obstet. 2016;293(1):125-31.

37. Ware JE Jr, Kosinski M, Gandek B, et al. The factor structure of the SF-36 health survey in 10 countries: results from the IQOLA Project. international quality of life assessment. J Clin Epidemiol. 1998;51(11):1159-65.

38. de Tayrac R, Alves A, Therin M. Collagen-coated vs noncoated low-weight polypropylene meshes in a sheep model for vaginal surgery. A pilot study. Int Urogynecol J Pelvic Floor Dysfunct. 2007;18(5):513-20.

39. Lo TS, Tan YL, Khanuengkitkong S, Dass AK, Cortes $\mathrm{EF}, \mathrm{Wu}$ PY. Assessment of collagen-coated anterior mesh through morphology and clinical outcomes in pelvic reconstructive surgery for pelvic organ prolapse. J Minim Invasive Gynecol. 2014;21(5):753-61.

40. Feola A, Endo M, Urbankova I, et al. Host reaction to vaginally inserted collagen containing polypropylene implants in sheep. Am J Obstet Gynecol. 2015;212(4):474 (e1-8).

41. Darzi S, Urbankova I, Su K, et al. Tissue response to collagen containing polypropylene meshes in an ovine vaginal repair model. Acta Biomater. 2016;39:114-23.

42. Cervigni M, Natale F, La Penna C, Saltari M, Padoa A, Agostini M. Collagen-coated polypropylene mesh in vaginal prolapse surgery: an observational study. Eur J Obstet Gynecol Reprod Biol. 2011;156(2):223-7.

43. Lo TS, Cortes EF, Wu PY, Tan YL, Al-Kharabsheh A, Pue LB. Assessment of collagen versus non collagen coated anterior vaginal mesh in pelvic reconstructive surgery: prospective study. Eur J Obstet Gynecol Reprod Biol. 2016;198:138-44.

44. Siniscalchi RT, Melo M, Palma PC, Dal Fabbro IM, Vidal Bde C, Riccetto CL. Highly purified collagen coating enhances tissue adherence and integration properties of monofilament polypropylene meshes. Int Urogynecol J. 2013;24(10):1747-54.

45. Cornwell KG, Zhang F, Lineaweaver W. Bovine fetal collagen reinforcement in a small animal model of hernia with component repair. J Surg Res. 2016;201(2):416-24.

46. Avila OR, Parizzi NG, Souza AP, Botini DS, Alves JY, Almeida SH. Histological response to platelet-rich plasma added to polypropylene mesh implemented in rabbits. Int Braz J Urol. 2016;42(5):993-8.

47. Liang R, Knight K, Barone W, et al. Extracellular matrix regenerative graft attenuates the negative impact of polypropylene prolapse mesh on vagina in rhesus macaque. Am J Obstet Gynecol. 2017;216(2):153e1.

48. Ergina PL, Barkun JS, McCulloch P, Cook JA, Altman DG, IDEAL Group. IDEAL framework for surgical innovation 2: observational studies in the exploration and assessment stages. BMJ. 2013;346:f3011. 